\title{
Ground state of trapped interacting Bose-Einstein condensates by an explicit imaginary-time algorithm
}

\author{
M. L. Chiofalo, ${ }^{1}$ S. Succi, ${ }^{2}$ and M. P. Tosi ${ }^{1}$ \\ ${ }^{1}$ Istituto Nazionale di Fisica della Materia and Classe di Scienze, Scuola Normale Superiore, I-56126 Pisa, Italy \\ ${ }^{2}$ Istituto Applicazioni Calcolo “M. Picone,"' Via del Policlinico 137, I-00161 Roma, Italy
}

(Received 20 April 2000)

\begin{abstract}
We show that an explicit time-marching method previously developed for the numerical study of the dynamics of Bose-Einstein condensates can be profitably adapted to the numerical determination of their ground state. After reduction to a one-dimensional model, we first reproduce and test known results on condensates in harmonic traps and then determine the ground state of a condensate in a harmonically bound optical lattice in the range of parameters which are relevant to existing experiments.
\end{abstract}

PACS number(s): 02.60.-x, 03.75.Fi, 32.80.-t, 42.50.-p

\section{INTRODUCTION}

The experimental realization of Bose-Einstein condensation (BEC) of trapped atomic vapors [1-3] has spurred a great deal of experimental, theoretical, and computational activity, all aimed at gaining a deeper insight into the fascinating behavior of this state of matter. Computational methods have been developed to analyze both the dynamical behavior and the ground-state properties of Bose-Einstein condensates, which are based on the solution of the timedependent and stationary Gross-Pitaevskii equation (GPE) $[4,5]$, respectively. Typically, these two scenarios are handled with distinct numerical techniques; implicit and/or explicit time-marching schemes for the former, and eigenvalue and/or variational solvers for the latter. However, it is well known that minimization procedures based on (fictitious) dynamics can often provide the most effective option also to compute ground-state properties of quantum systems $[6,7]$.

Besides the intrinsic interest of computing the ground state of BEC matter per se, the availability of ground-state numerical solvers is also important for BEC dynamics because the dynamic behavior of BEC matter can be very sensitive to initial conditions.

The implementation of numerical methods for groundstate calculations on one-dimensional systems is a highly developed subject in computational condensed matter [7]. These methods rely mostly on clever techniques to diagonalize the Hamiltonian which, in some cases, may even lead to exact solutions. However, owing to the nonlinearity built in the GPE, the computation calls for the solution of a whole sequence of linear(ized) problems, each requiring its own diagonalization. Under these conditions, a dynamic procedure may be more efficient.

Many such dynamic (minimization) procedures are again available from Monte Carlo simulation techniques. For example, the basic idea of diffusion Monte Carlo [8] is to formulate the Schrödinger equation in imaginary time so as to turn it into a real-time diffusion equation whose steady-state yields the ground-state of the corresponding quantum system. For the case of single-particle equations, such as the GPE, the solution of the diffusion problem does not even require any expensive Monte Carlo procedure, but can be carried out by means of standard grid methods. Among these, perhaps the most popular choice is finite differencing of space variables as combined with semi-implicit (CranckNicholson) time marching [9]. The key points of semiimplicit methods are (i) freedom from stringent stability constraints on the time-step size and (ii) the attendant capability to march in large steps to steady state. The price for this is the need to solve a linear algebraic problem at each time step, in principle a rather expensive computational task. In this paper we present an alternative method based on the use of explicit algorithms. We shall not make any point of superior efficiency as compared with implicit methods (such a point can be made for genuinely time-dependent methods) but just highlight the simplicity, flexibility, and ease-of-use afforded by explicit methods at a reasonable computational efficiency.

\section{MODEL}

In this paper we shall be interested in effectively onedimensional (1D) BEC systems. Despite their mathematical simplicity, these systems bear nonetheless a significant physical interest, mainly in view of experiments on the transport behavior of condensates in elongated optical traps which are periodic in only one direction of space [10]. Such optical lattices can also give access to the spin degrees of freedom, which are indeed frozen in magnetic-type traps.

At zero temperature the dynamics of a dilute trapped Bose condensate is well described by the time-dependent GPE for the condensate wave function $\Psi(\mathbf{r}, t)[4,5]$

$$
i \hbar \frac{\partial \Psi(\mathbf{r}, t)}{\partial t}=\left(-\frac{\hbar^{2}}{2 M} \nabla_{\mathbf{r}}^{2}+U_{\mathrm{ext}}(\mathbf{r})+U_{I}|\Psi(\mathbf{r}, t)|^{2}\right) \Psi(\mathbf{r}, t) .
$$

Here, $M$ is the atomic mass, $U_{1}=4 \pi \hbar^{2} a N / M$ is the coupling strength, and $U_{\text {ext }}$ is the external potential, $a$ being the scattering length, and $N$ the number of particles in the condensate. In what follows $U_{\text {ext }}(\mathbf{r})$ is due either to a harmonic magnetic trap or to an optical lattice. In the former case

$$
U_{\mathrm{ext}, h}(r, z)=\frac{1}{2} M \omega^{2}\left(r^{2}+\epsilon^{2} z^{2}\right),
$$


$\epsilon=\omega_{z} / \omega$ being the aspect ratio. In the latter case [10],

$$
U_{\text {ext }, l}(r, z)=U_{l}^{0}\left[1-\exp \left(-r^{2} / r_{l b}^{2}\right) \cos ^{2}(2 \pi z / \lambda)\right],
$$

where $U_{l}^{0}$ is the well depth, $r_{l b}$ is the transverse size, and the wavelength $\lambda$ yields the lattice period $d=\lambda / 2$. Finally, the normalization condition for the wave function is $\int|\Psi(\mathbf{r}, t)|^{2} d \mathbf{r}=1$.

An effective model for the mean-field interaction in the 1D GPE can be derived by requiring that the chemical potential $\mu$ in $1 \mathrm{D}$ be equal to the chemical potential of the three-dimensional (3D) system. This model has been used in the context of harmonic magnetic traps $[11,12]$ and has been shown to be best performing in the case of optical lattices, where the dynamical simulation of the emission of coherent matter wave pulses from a condensate under the action of gravity is found to yield quantitative agreement with full 3D calculations [13].

In brief, the reduction to a 1D model turns Eq. (1) into the following 1D equation:

$$
i \hbar \frac{\partial \psi(z, t)}{\partial t}=\left(-\frac{\hbar^{2}}{2 M} \frac{\partial^{2}}{\partial z^{2}}+u_{\mathrm{ext}}(z)+u_{I}|\psi(z, t)|^{2}\right) \psi(z, t) .
$$

Here $u_{\mathrm{ext}}(z)$ is the external one-dimensional potential and $u_{I}=4 \pi \hbar^{2} \widetilde{a} N / M, \widetilde{a} \equiv a \gamma$ being a renormalized coupling parameter with the dimensions of an inverse length.

In the case of harmonic confinement $\left[u_{\mathrm{ext}, h}(z)\right.$ $\left.=M \omega^{2} \epsilon^{2} z^{2} / 2\right]$ one obtains $[11,12]$

$$
\gamma_{h}=\left(\frac{5}{2 \pi}\right)^{3 / 5}\left(24 \pi \epsilon N a / S_{l}\right)^{-2 / 5} / S_{l}^{2},
$$

with $S_{1}=(\hbar / 2 M \omega)^{1 / 2}$. In the case of optical lattice confinement $\left[u_{\mathrm{ext}, l}(z)=U_{l}^{0} \sin ^{2}(2 \pi z / \lambda)\right]$ we find [13]

$$
\gamma_{l}=\frac{1}{\pi r_{l b}^{2}}+\frac{U_{l}^{0}-\mu}{4 E_{R}} \frac{1}{\epsilon a N} I(\mu),
$$

$E_{R}=h^{2} /\left(2 M \lambda^{2}\right)$ being the recoil energy. The explicit expression for the positive quantity $I(\mu)$ in Eq. (6) is

$$
I(\mu)=\int_{0}^{f(\mu)} w \tan (w / 2) d w>0,
$$

with $f(\mu)=\arccos \left[1-2 \mu / U_{l}^{0}\right]$.

\section{NUMERICAL METHOD}

Our numerical method is based on an imaginary-time formulation of the effective 1D GPE (4). By a Wick rotation

$$
t \rightarrow \tau=-i t
$$

the 1D GPE turns into a diffusion equation with absorption/ emission due to the potential term. In solving this equation we use an explicit synchronous Visscher scheme [14]. Since the details of this scheme have been published elsewhere [15], in the following we shall only recall the basic steps. The method evolves the real and imaginary parts of the wave function $\psi=\mathfrak{R}+i \mathfrak{I}$ according to the relations $\partial_{t} \mathfrak{R}=\mathcal{H} \mathfrak{I}$ and $\partial_{t} \mathfrak{I}=-\mathcal{H} \mathfrak{R}$, where $\mathcal{H}=\mathcal{T}+\mathcal{V}$ is the Hamiltonian consisting of kinetic plus potential energy operators. $\mathcal{T}=\left(\hbar^{2} /\right.$ $2 M)\left(\partial^{2} / \partial z^{2}\right)$ and $\mathcal{V}=u_{\text {ext }}+g|\psi|^{2}$, with $g=8 \pi \tilde{a} N$. Under the Wick rotation, this system turns into

$$
\partial_{\tau \mathfrak{R}}=-\mathcal{H} \mathfrak{R}
$$

and

$$
\partial_{\tau} \mathfrak{I}=-\mathcal{H} \mathfrak{I}
$$

showing that the real and imaginary parts of the wave function evolve independently according to a diffusion equation with a (nonlinear) source and/or sink term.

The diffusion system can be evolved according to the same scheme previously used for the real-time evolution equations [15]. This consists of a centered finite-differencing for the spatial variable and an explicit time marching for the variable $\tau$. The result is the following finite-difference scheme:

$$
\mathfrak{R}_{j}^{k+1}=\mathfrak{R}_{j}^{k-1}-2 \Delta \tau\left(\mathcal{T}+\mathcal{V}^{k}\right) \mathfrak{R}_{j}^{k}
$$

and

$$
\mathfrak{I}_{j}^{k+1}=\mathfrak{I}_{j}^{k-1}-2 \Delta \tau\left(\mathcal{T}+\mathcal{V}^{k}\right) \mathfrak{I}_{j}^{k},
$$

where $\mathcal{T} \psi_{j}^{k} \equiv\left(\psi_{j+1}^{k}-2 \psi_{j}^{k}+\psi_{j-1}^{k}\right) /\left(\Delta_{z}\right)^{2}$ and $\mathcal{V}^{k} \psi_{j}^{k} \equiv V_{j}^{k} \psi_{j}^{k}$.

The scheme is initiated with a single Euler-forward step [16] from $k=0$ to $k=1$ using Eq. (11) with a time step $\Delta \tau: \mathfrak{R}_{j}^{1}=-\Delta \tau\left(\mathcal{T}+\mathcal{V}^{0}\right) \mathfrak{R}_{j}^{0}$ and $\mathfrak{I}_{j}^{1}=-\Delta \tau\left(\mathcal{T}+\mathcal{V}^{0}\right) \mathfrak{I}_{j}^{0}$. Once level $k=1$ is available, the time-marching can proceed in steps of $2 \Delta \tau$ as indicated in Eqs. (11) and (12). We summarize in the following the main properties of this numerical scheme.

\section{A. Unitarity and relaxation}

The real-time formulation of this algorithm can be shown [15] to preserve unitarity at each discrete time $t_{k}$, provided that (i) the discrete probability density at the space location (j) and time $k+1$ is defined as $P_{j}^{k+1} \equiv \mathfrak{R}_{j}^{k+1} \mathfrak{R}_{j}^{k}+\mathfrak{I}_{j}^{k+1} \mathfrak{I}_{j}^{k}$, and (ii) the boundary conditions are such as to annihilate surface terms. This is indeed the case of our applications, since we impose periodicity along $z$. For the imaginary-time version, the norm $n$ is obviously decaying in time due to the potential term. This decay can be expressed as follows:

$$
n(\tau) \equiv \int|\psi(z, \tau)|^{2} d z=\sum_{l \geqslant 0} p_{l}(0) e^{-\omega_{l} \tau},
$$

where $p_{l}(0)=p_{l}(\tau)$ is the projection upon the $l$ th eigenstate of energy $E_{l}$. The quantity $e(\tau) \equiv \int \psi^{*} \mathcal{H} \psi d z$ follows a similar decay:

$$
\int \psi^{*} \mathcal{H} \psi d z=\frac{1}{n(\tau)} \sum_{l \geqslant 0} p_{l}(0) E_{l} e^{-E_{l} \tau / \hbar} .
$$

It is an easy matter to show that for a given spectrum of eigenvalues $\left\{E_{l}\right\}$, the average energy $\langle E(\tau)\rangle=e(\tau) / n(\tau)$ decays monotonically in time till the steady state is reached: $n(\tau) \rightarrow p_{0} e^{-E_{0} \tau / \hbar}, \quad e(\tau) \rightarrow p_{0} E_{0} e^{-E_{0} \tau / \hbar}$, and $\langle E(\tau)\rangle \rightarrow E_{0}$. 
The ground-state energy can then be read off from the steady-state value of the specific energy as $\tau \rightarrow \infty$.

As a final comment, we notice that in order to maintain a reasonable signal-to-noise ratio it is a good practice to restore unitarity $[n(\tau)=1]$ every few steps during the simulation. In actual practice, we have found it convenient to renormalize the wave function at each time step. This implies a small computational overhead but ensures optimal signal-tonoise ratio.

\section{B. Stability}

Following a standard procedure [17], in order to appraise the stability of the discrete evolution operator (11) and (12), we decompose the solution in plane waves $\exp \left[i\left(\chi z_{j}-\Omega t_{k}\right)\right]$ and require that the resulting dispersion relation does not give rise to any time-growing (spurious) solutions.

This yields a stability limit in the following general form:

$$
\Delta \tau\left(\left|C_{1} D /(\Delta z)^{2}+C_{2} V_{M} / \hbar\right|\right)<1,
$$

where $D \equiv \hbar / 2 M$ is the quantum diffusivity, $V_{M}$ the maximum value of the potential, and $C_{1}, C_{2}$ two numerical constants which for the case in point take the values $C_{1}=2$ and $C_{2}=1 / 2$. The above relation identifies the largest acceptable dimensionless time step $\Delta \tau_{c}$ as

$$
\Delta \tau_{c}=\frac{(\Delta z)^{2}}{C_{1} D+C_{2}\left(V_{M} / \hbar\right)(\Delta z)^{2}} .
$$

This expression shows that interaction potentials below the numerical threshold $V_{M}<V_{\Delta} \equiv\left(C_{1} / C_{2}\right) L$ do not affect the standard Courant-Friedrichs-Lewy condition $\Delta \tau \sim(\Delta z)^{2}$ for the diffusion equation. This is a severe bound since it forces the time step to decrease with the square of the grid spacing. ${ }^{1}$ Above $V_{\Delta}$, the time step is basically controled by the potential alone, $\Delta \tau<\hbar /\left(C_{2} V_{M}\right)$ independently of the mesh size. This regime implies limitations on the physical parameters characterizing the interaction potential and in particular forces the time step to scale inversely with the number of bosons.

It is readily checked that for typical values of current experimental interest, i.e., $N \sim 10^{6}$ and $\widetilde{a} \sim 10^{-5}$ in dimensionless units (see Sec. IV) the time step in the numerical simulation is diffusion limited. In fact, leaving the external potential aside for simplicity, the condition $V_{M}<V_{\Delta}$ yields

$$
N<N_{\max }=\frac{1}{2 \pi} \frac{S_{l}^{2}}{(\Delta z)^{2}} \frac{1}{\widetilde{a} S_{l}},
$$

where $S_{l}$ is the typical length scale (see Sec. IV). For the typical value $\Delta z / S_{l} \sim 10^{-2}$, this yields $N_{\max } \sim 10^{8}$. In order to secure a further margin of stability, we have adopted an empirical safety factor by keeping the actual time step consistently below the marginal threshold, typically by a factor of about 0.2 .

\footnotetext{
${ }^{1}$ Such quadratic constraint can be brought down to a linear dependence by using lattice kinetic methods [18]. However, the latter are not straightforwardly extended to non-Cartesian geometries.
}

\section{SIMULATION RESULTS}

We use the numerical scheme presented in the previous section to calculate the ground state of 1D BEC's trapped in two different geometries. We first recover known results on condensates in harmonic potentials, which are related to the very first experiments on BEC [1-3]. We then turn to an original problem, which refers to the ground state of a condensate in an optical lattice, related to the experiment of Anderson and Kasevich [10].

The whole series of runs refers to atoms of ${ }^{87} \mathrm{Rb}$, whose scattering length is $a=110$ Bohr radii. As to the optical lattice parameters, we take $\lambda=850 \mathrm{~nm}$ and $r_{l b}=80 \mu \mathrm{m}$ from the experiment. The typical height of the barrier in the experiment is $U_{l}^{0}=1.4 E_{R}$. Finally, we adopt the units $S_{l}$ $=\sqrt{\hbar / 2 M \omega}, S_{E}=\hbar \omega$, and $S_{t}=1 / \omega$ for length, energy, and time, to turn Eq. (4) into a dimensionless form.

\section{A. Harmonic potential}

For simplicity, we treat here the case of an isotropic harmonic potential $u_{\mathrm{ex}, h}$ with $\epsilon=1$. Besides their usefulness as test cases, there is new experimental activity in producing spherical traps [19]. Isotropic geometries are theoretically interesting in view of the effects that the reduced avalability of states has on the damping mechanism of the condensate and noncondensate excitations [19]. We choose the initial guess for the imaginary-time evolution in the form of a nonminimal Gaussian wave packet $\psi(z) \propto \exp [-M \widetilde{\omega}(z$ $\left.\left.-z_{0}\right)^{2} /(2 \hbar)\right]$ with $\widetilde{\omega}=\omega / 2$. In the absence of the nonlinear term in Eq. (4), such a wave packet would relax to the ground state of the harmonic oscillator, characterized by $\widetilde{\omega}$ $=\omega$. As self-interactions are switched on, the ground state takes the form of an "interpolate" between a noninteracting Gaussian (in the tail of the distribution) and an inverse parabolic profile in the trap center [20]. The physical picture is that in the interaction-dominated regime the neglect of the kinetic energy term in Eq. (4) yields the well-known Thomas-Fermi expression

$$
\psi_{\mathrm{TF}}(z)=\frac{\mu-u_{\mathrm{ext}, h}(z)}{u_{I}},
$$

the chemical potential $\mu$ being given by the normalization condition on the wave function. The Gaussian tails in the distribution derive from the Laplacian in Eq. (4), which becomes dominant over the interaction potential term.

The ground state has been computed for a series of values of $N=1 \times 10^{4}, 5 \times 10^{4}, 1 \times 10^{5}, 2 \times 10^{5}, 5 \times 10^{5}, 1 \times 10^{6}$, and $2 \times 10^{6}$, which correspond to increasing strength of the interactions. In all cases a 7391-point grid, extending from $-18<\zeta<18$ has been used, $\zeta$ being the dimensionless coordinate $\zeta=z / S_{l}$. This grid corresponds to $\Delta \zeta=5 \times 10^{-3}$. The typical dimensionless time step is $\Delta \tau=1 \times 10^{-6}$, which is below the diffusive stability threshold $\Delta \tau_{c}=0.5(\Delta \zeta)^{2} \sim 1.2$ $\times 10^{-5}$.

The resulting steady-state density profile $|\psi(z)|^{2}$ is shown in Fig. 1 for the whole sequence of $N$ values. The progressive broadening of the wave function with increasing number of atoms in the condensate is in accord with the physical picture mentioned above. As a convergence criterion, the imaginarytime simulation is stopped upon steadiness in the fifth digit 


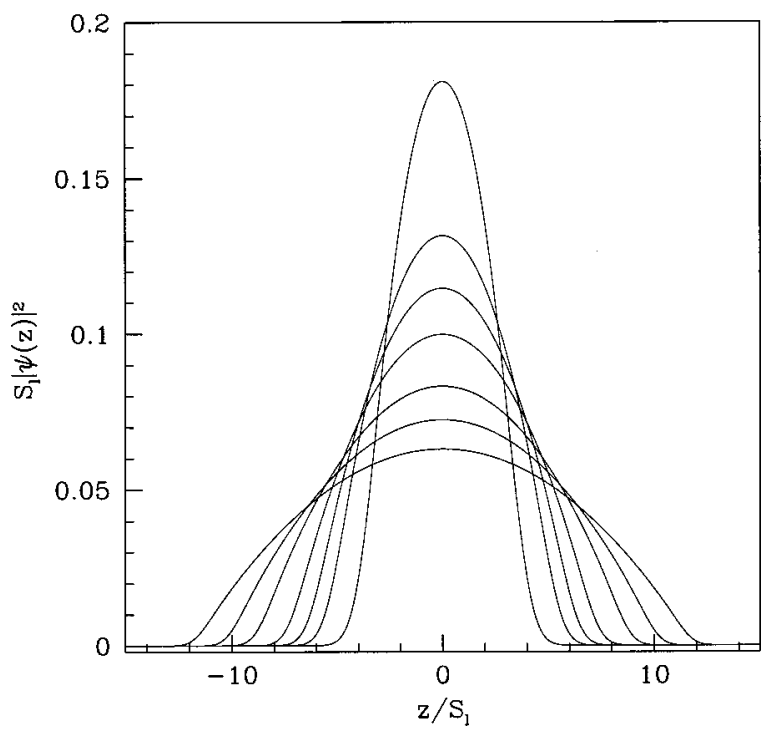

FIG. 1. Condensate in harmonic trap. Density profiles $S_{l}|\psi(z)|^{2}$ at $t=0$ for $a=110 \mathrm{Bohr}$ radii and various values of $N$. From top to bottom in the central part of the figure: $N=1 \times 10^{4}, 5 \times 10^{4}, 1$ $\times 10^{5}, 2 \times 10^{5}, 5 \times 10^{5}, 1 \times 10^{6}$, and $2 \times 10^{6}$.

of the average total energy $\langle E(\tau)\rangle$. This typically requires a number of time steps of the order one million. A typical energy relaxation pattern is shown in Fig. 2 for different values of $N$. The computed values of the ground-state energy are reported in Table I together with the values of the Thomas-Fermi chemical potential $\mu_{T F}$ at the corresponding value of $N$. From the table we observe that, as expected, the computed values are very close to the Thomas-Fermi approximation and consistently above it.

The above results are consistent with earlier numerical studies of the ground state of harmonically confined BoseEinstein condensates [21,22]. Here we are mainly concerned,

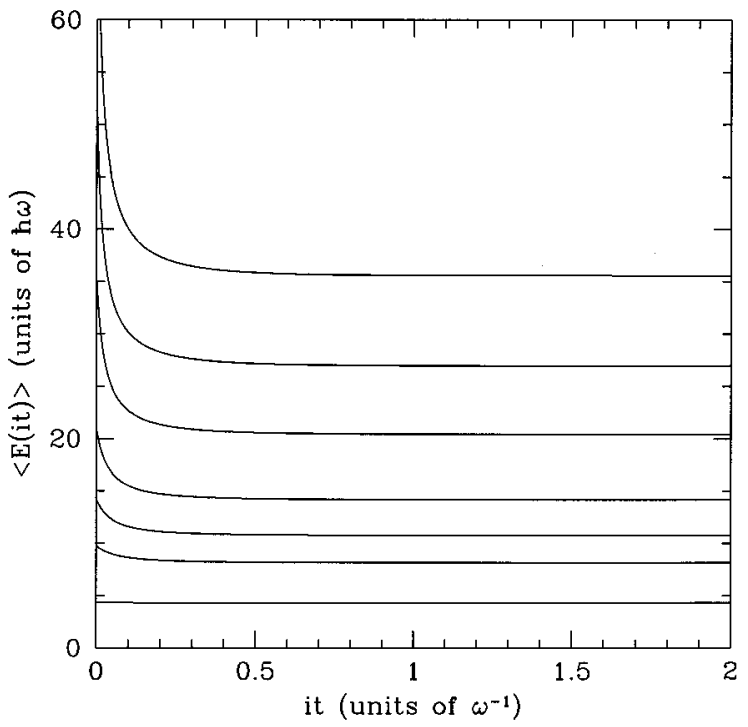

FIG. 2. Condensate in harmonic trap. Evolution of the average total energy $\langle E\rangle$ in imaginary time for various values of $N$. From bottom to top: $N=1 \times 10^{4}, 5 \times 10^{4}, 1 \times 10^{5}, 2 \times 10^{5}, 5 \times 10^{5}, 1$ $\times 10^{6}$, and $2 \times 10^{6}$.
TABLE I. Condensate in harmonic trap. Ground-state energy $\mu / \hbar \omega$ and $3 \mathrm{D}$ chemical potential $\mu_{\mathrm{TF}} / \hbar \omega$ in the Thomas-Fermi approximation for various values of $N$.

\begin{tabular}{crr}
\hline \hline$N$ & $\mu / \hbar \omega$ & $\mu_{\mathrm{TF}} / \hbar \omega$ \\
\hline $1 \times 10^{4}$ & 4.314 & 4.263 \\
$5 \times 10^{4}$ & 8.149 & 8.116 \\
$1 \times 10^{5}$ & 10.738 & 10.710 \\
$2 \times 10^{5}$ & 14.159 & 14.130 \\
$5 \times 10^{5}$ & 20.422 & 20.386 \\
$1 \times 10^{6}$ & 26.949 & 26.899 \\
$2 \times 10^{6}$ & 35.572 & 35.493 \\
\hline \hline
\end{tabular}

however, with an accurate determination of the ground state as the initial state for simulation studies of dynamical behavior. Therefore, as an independent check of steadiness we use the ground state $\psi_{0}(z)$ computed by the imaginary-time simulation as an initial state for a real-time evolution, the idea being that a genuine ground state should evolve in real time like a purely oscillating mode at frequency $\omega_{0}=\mu / \hbar$ with no change in the density profile, namely,

$$
\psi(z, t)=\psi_{0}(z) e^{-i \mu t / \hbar} .
$$

The ground-state energy is then read off from the time trace of the real (imaginary) part of the wave function at any given spatial location $z$. Such periodic oscillations are reproduced to an outstanding degree of accuracy in the temporal patterns shown in Fig. 3 for the cases $N=10^{4}, 10^{5}$, and $10^{6}$. As a further test, we check that the density profile remains independent of time. This is again true to a high accuracy in our results, as shown in Fig. 4 in the case $N=2 \times 10^{5}$. The main body of the figure shows the ground-state amplitude (and the phase pattern $\phi$ ) as obtained from the imaginary-time simu-

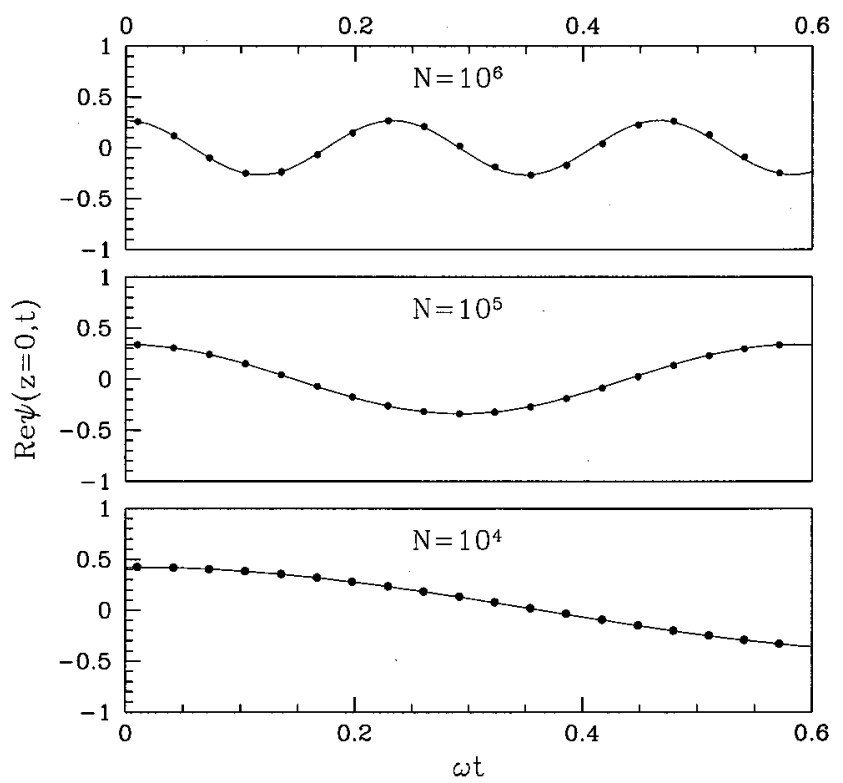

FIG. 3. Condensate in harmonic trap. Time evolution of $\mathfrak{R} \psi(z$ $=0, t)$ : from bottom to top, the cases $N=10^{4}, 10^{5}$, and $10^{6}$ are shown. The points are the theoretical prediction $\mathfrak{R} \psi(z=0, t)$ $=|\psi(z=0,0)| \cos (\mu t)$, with $\mu=4.314,10.738$, and $26.949 \hbar \omega$ for each case as in Table I. 


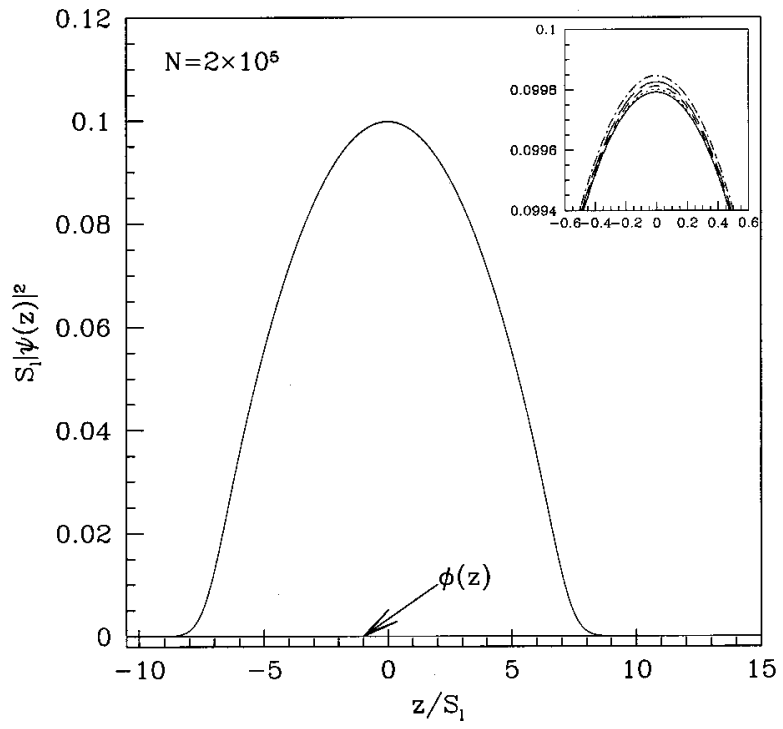

FIG. 4. Condensate in harmonic trap. Density profiles $S_{l}|\psi(z)|^{2}$ at different times for $N=2 \times 10^{5}$. From bottom to top, as zoomed in the inset: $t=0,12,24,36$, and $48 \mathrm{~ms}$. The (flat) pattern of the phase $\phi(z)$ at $t=0$ is marked by an arrow.

lation. The subsequent real-time evolved density profile is shown in the inset on a greatly magnified scale in order to display otherwise visually unappreciable changes.

\section{B. Optical lattice potential}

In the present section we calculate the BEC ground state in the periodic optical potential $u_{\text {ext }, l}$, to which a harmonic confinement $M \omega^{2} z^{2} / 2$ is superposed. The calculation is simplified by the fact that the chemical potential of the system in the harmonic trap is of the order of the harmonic energy $\hbar \omega$ ( $\mu=\hbar \omega / 2$ in the noninteracting case), while that of the system in the optical lattice is of the order of the recoil energy $E_{R}$ [13]. Since the ratio of the harmonic-to-recoil energy is $\hbar \omega / E_{R} \simeq 1 / 464$ for the present range of parameters, we may neglect the renormalization of the scattering length coming from the superposed harmonic confinement and use the result given in Eq. (6).

The initial guess for the imaginary-time evolution is then chosen as

$$
\psi(z)=A \exp \left[-M \widetilde{\omega} z^{2} / 2 \hbar\right] \sum_{l} \exp \left[-M \omega_{h a}(z-l d)^{2} / 2 \hbar\right],
$$

which is a sum of Gaussian profiles centered on the lattice sites, with a width dictated by the harmonic approximation to each lattice well [corresponding to the frequency $\omega_{h a}$ in Eq. (20)]. Due to the harmonic confinement, an overall nonminimal Gaussian envelope shapes the profile. In Eq. (20) $A$ is a normalization factor and $l$ labels the occupied sites.

Figure 5 shows the density profiles which are obtained at fixed barrier height $U_{l}^{0}=1.4 E_{R}$ for increasing values of $N$ $=10^{4}, 10^{5}$, and $3 \times 10^{5}$ from the bottom to the top panel. The corresponding values of the parameter $\gamma S_{l}^{2}$ are $\gamma S_{l}^{2}$ $=5.7 \times 10^{-3}, 2.1 \times 10^{-3}$, and $1.3 \times 10^{-3}$ [13]. Peaks show up in correspondence to the lattice sites, which are counted by the adimensional variable $2 z / \lambda$. As to the effect of the

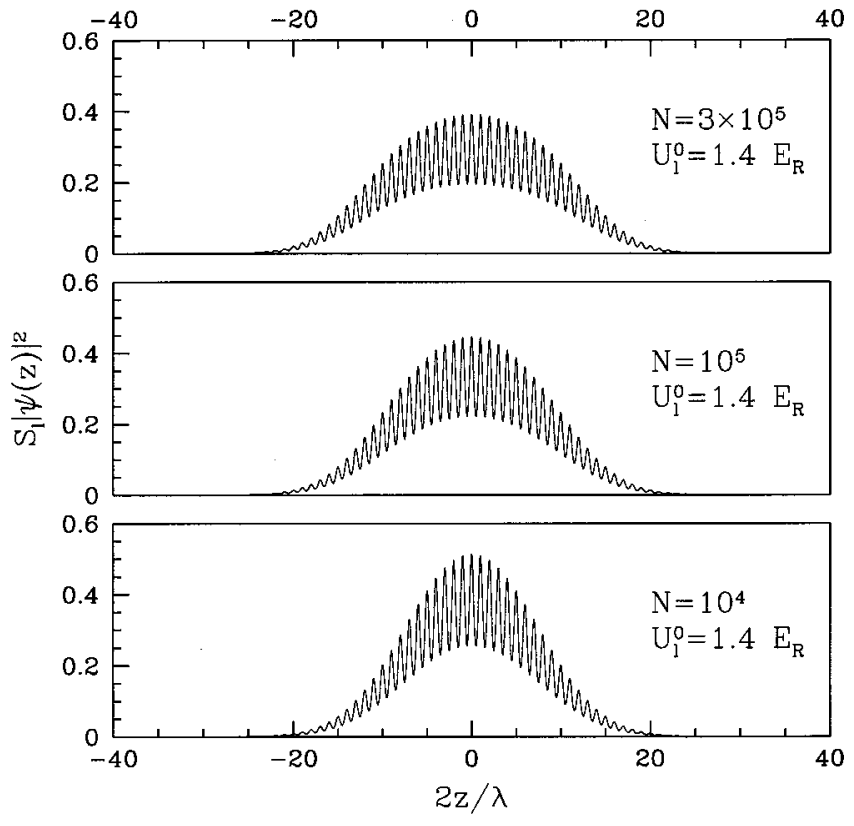

FIG. 5. Condensate in optical lattice plus harmonic trap. Density profiles of a condensate with different numbers of atoms in an optical lattice with $U_{l}^{0}=1.4 E_{R}$. From bottom to top panel: $N=10^{4}$, $10^{5}$, and $3 \times 10^{5}$. The abscissa is scaled with the lattice period $\lambda / 2$ (see text).

interactions, a similar trend as in Fig. 1 is visible in the overall shape with increasing $N$. The calculated values of the chemical potential are $\mu=0.644 E_{R}$ for $N=10^{4}, \mu$ $=0.648 E_{R}$ for $N=10^{5}$, and $\mu=0.652 E_{R}$ for $N=3 \times 10^{5}$. It should be remarked that, even though three digits are reliable from the point of view of the numerical simulation, this is not really the case because of the physical approximation used in calculating $\gamma_{l}$.
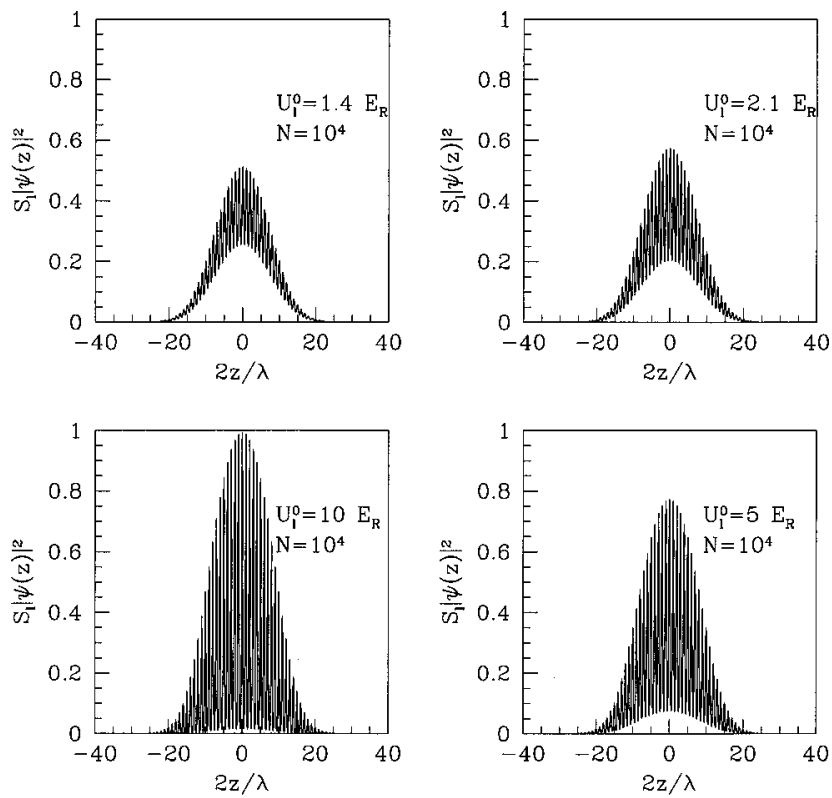

FIG. 6. Condensate in optical lattice plus harmonic trap. Density profiles of a condensate with $N=10^{4}$ atoms in an optical lattice with different barrier heights. Clockwise from the top-left panel: $U_{l}^{0}=1.4,2.1,5$, and $10 E_{R}$. 


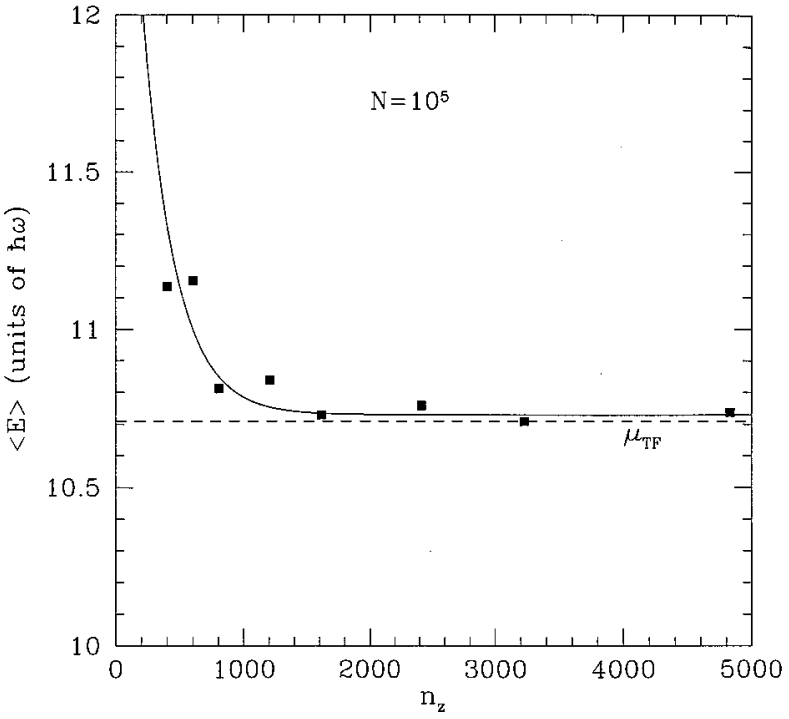

FIG. 7. The average total energy $\langle E\rangle$ for a condensate with $N$ $=10^{5}$ atoms in a harmonic trap, as obtained after convergence in the imaginary-time simulation, is plotted as a function of the number of grid points. The dashed line shows the corresponding value of the Thomas-Fermi chemical potential.

Figure 6 displays how the density profiles change on increasing the barrier height at fixed $N=10^{4}$. Clockwise from the top-left panel, the results for the cases $U_{l}^{0}=1.4,2.1,5$, and $10 E_{R}$ are reported. The corresponding values of $\gamma S_{l}^{2}$ are $\gamma S_{l}^{2}=5.7 \times 10^{-3}, 6.8 \times 10^{-3}, 9.6 \times 10^{-3}$, and $1.3 \times 10^{-2}$. The calculated values of the chemical potential are $\mu$ $=0.923 E_{R}$ for $U_{l}^{0}=2.1 E_{R}, 1.835 E_{R}$ for $U_{l}^{0}=5 E_{R}$, and $2.869 E_{R}$ for $U_{l}^{0}=10 E_{R}$. The figure shows that an increase in the well depth yields progressive localization of the portions of condensate in each well. We expect that the original condensate will eventually fragment into pieces, their relative phases becoming uncorrelated $[23,24]$. The analysis of such an issue will be the subject of future work.

\section{Computational performance}

The code takes about $0.4 \mu$ s per grid point and time step on a PC (double Pentium II $450 \mathrm{MhZ}$ ), corresponding to about $0.5 \mathrm{CPU}$ hours for a fully converged (fifth digit) ground state. This figure can be considerably reduced by lowering the accuracy requirements. In fact, since the time step scales roughly like the square of the mesh spacing, halving the spatial resolution brings savings in CPU time by a factor of 8 .

A quantitative feeling for the dependence of accuracy on the number of grid points is conveyed in Fig. 7, where the ground-state energy of the condensate in a harmonic trap is shown as a function of the number of grid points in the simulation, the dashed line indicating the corresponding $\mu_{\mathrm{TF}}$ as a reference. From this figure we see that the convergence to the asymptotic value is basically quadratic, in accord with earlier findings in time-dependent applications [15]. In particular, it is seen from Fig. 7 that about 1000 grid points are sufficient to ensure an accuracy within a few percent. Such a simulation requires a few minutes of CPU time on a midrange workstation.

\section{SUMMARY}

We have shown that an explicit time-marching method combined with the well-known concept of evolution in imaginary time can be profitably adapted to the numerical determination of the ground state of dilute Bose-Einstein condensates, which obey a nonlinear Schrödinger equation. We have given two examples of one-dimensional systems which are relevant to experiments in harmonic traps and optical lattices. Such simulations can be carried out in a few minutes of CPU time on a midrange workstation with an accuracy of a few percent.
[1] M. H. Anderson, J. R. Ensher, M. R. Matthews, C. E. Wieman, and E. A. Cornell, Science 269, 198 (1995).

[2] K. B. Davis, M. O. Mewes, M. R. Andrews, N. J. van Druten, D. S. Durfee, D. M. Kurn, and W. Ketterle, Phys. Rev. Lett. 75, 3969 (1995).

[3] C. C. Bradley, C. A. Sackett, and R. G. Hulet, Phys. Rev. Lett. 78, 985 (1997)

[4] E. P. Gross, Nuovo Cimento 20, 451 (1961).

[5] L. P. Pitaevskii, Zh. Eksp. Teor. Fiz. 40, 646 (1961) [Sov. Phys. JETP 13, 451 (1961)].

[6] See, e.g., T. Pang, An Introduction to Computational Physics (Cambridge University Press, Cambridge, England, 1997).

[7] J. M. Thijssen, Computational Physics Cambridge (Cambridge University Press, Cambridge, England, 1998).

[8] M. Kalos, D. Levesque, and L. Verlet, Phys. Rev. A 9, 2178 (1974); D. M. Ceperley, G. Chester, and M. Kalos, Phys. Rev. B 16, 3081 (1977); D. M. Ceperley and M. Kalos, in Monte Carlo Methods in Statistical Physics, edited by K. Binder (Springer, Berlin, 1986), p. 94.

[9] M. J. Holland and J. Cooper, Phys. Rev. A 53, R1954 (1996).
[10] B. P. Anderson and M. A. Kasevich, Science 281, 1686 (1998).

[11] J. E. Williams, Ph.D. thesis, University of Colorado, Boulder, 1999 (unpublished).

[12] J. Schneider and A. Schenzle, Appl. Phys. B: Lasers Opt. 69, 353 (1999).

[13] M. L. Chiofalo, M. P. Tosi, Phys. Lett. A (to be published).

[14] P. B. Visscher, Comput. Phys. 5, 596 (1991).

[15] M. M. Cerimele, M. L. Chiofalo, F. Pistella, S. Succi, and M. P. Tosi, Phys. Rev. E 62, 1382 (2000).

[16] D. Potter, Computational Physics (Wiley, London, 1977).

[17] A. R. Mitchell and D. F. Griffiths, The Finite Difference Method in Partial Differential Equations (Wiley, New York, 1980).

[18] S. Succi, Int. J. Mod. Phys. C 9, 1577 (1998); Phys. Rev. E 53, 1969 (1996).

[19] E. Hodby, G. Hechenblaikner, O. M. Maragò, J. Arlt, S. Hopkins, and C. J. Foot, e-print cond-mat/0004015.

[20] M. J. Holland, D. S. Jin, M. L. Chiofalo, and J. Cooper, Phys. Rev. Lett. 78, 3801 (1997).

[21] M. Edwards and K. Burnett, Phys. Rev. A 51, 1382 (1995); M. 
Edwards, R. J. Dodd, C. W. Clark, P. A. Ruprecht, and K. Burnett, ibid. 53, R1950 (1996).

[22] F. Dalfovo and S. Stringari, Phys. Rev. A 53, 2477 (1996).
[23] M. L. Chiofalo, S. Succi, and M. P. Tosi, Phys. Lett. A 260, 86 (1999).

[24] M. L. Chiofalo, M. Polini, and M. P. Tosi, Eur. Phys. J. D (to be published). 